\title{
Perfil das reclamações registradas acerca de roedores do Distrito Federal, Brasil
}

\section{Profile of registered complaints about rodents of the Federal District, Brazil}

\author{
Paulo Vinicius Neves Fernandes, ${ }^{* *}$ Lívia Thayane da Silva Cunha, ${ }^{* *}$ Debora Marcolino Silva, ${ }^{* \star}$ \\ Frederico Tôrres Braz, ${ }^{* *}$ Ligia Cantarino****
}

\begin{abstract}
Resumo
Os animais sinantrópicos, dentre eles os roedores, contituem problema de saúde pública em áreas urbanizadas. Foi realizado levantamento das reclamações sobre roedores feitas para o setor de animais sinantrópicos da Diretoria de Vigilância Ambiental do Distrito Federal no intervalo entre 2014 e 2016. As solicitações atingiram o número de 10.232, sendo 4.429 em 2014 , 4.368 e 1.429 nos respectivos anos subsequentes. A queda expressiva é decorrente da ausência de telefone no Órgão, se tratando da forma de comunicação mais utilizada para execução das solicitações. Quando relacionados a população das Regiões Administrativas com a quantidade de reclamações, houve relação, mas o mesmo não ocorreu com renda per capita e grau de escolaridade. 6.902 ocorrências foram em residências, justificadas pela população ansiando por desratização gratuita. Houve 11.901 casos confirmados de leptospirose no Brasil nos anos estudados. Destes, 82 ocorreram no DF. Mesmo que maiores os valores utilizados em programas de controle de roedores quando comparados a despesas com zoonoses transmitidas por esses animais, sendo custos diretos médicos, diretos não médicos, indiretos não mensuráveis, o bem-estar da sociedade deve ser destacado.
\end{abstract}

Palavras-chave: Controle de Roedores. Animais Sinantrópicos. Vigilância Ambiental.

\begin{abstract}
Synanthropic animals, including rodents, contain the public health problem in urbanized areas. A survey of rodent complaints made to the synanthropic animal sector of the Federal District Environmental Surveillance Directorate was carried out between 2014 and 2016. The occurrences reached 10,232, with 4,429 in 2014, 4,368 and 1,429 in subsequent years. The significant drop is due to the absence of a telephone in the Agency, being the most used form of communication for executing executions. When related to the population of the Administrative Regions with the number of complaints, there was a relationship, but the same did not occur with per capita income and education level. 6,902 occurrences were in homes, justified by the population yearning for free rat removal. There were 11,901 confirmed cases of leptospirosis in Brazil in the years studied. Of these, 82 occurred in the DF. Even though the values used in rodent control programs are higher when compared to expenses with zoonoses transmitted by these animals, being direct medical costs, non-medical direct costs, non-measurable indirect costs, society's well-being must be highlighted.
\end{abstract}

Keywords: Rodent Control. Synanthropic Animals. Environmental Surveillance.

\section{Introdução}

Os animais sinantrópicos, dentre eles os roedores, constituem um problema de saúde pública em áreas urbanizadas. 0 crescimento populacional e a precariedade de acesso ao saneamento básico, da drenagem deficiente de águas pluviais, da ausência ou irregularidade da coleta de resíduos sólidos propicia condições ideais à ligação comensal dos roedores com o homem num processo de sinantropia. Esta proximidade predispõe a população a diversos tipos de doenças, dentre elas, a leptospirose e hantavirose (BRASIL, 2002).

São três as espécies mais comuns de roedores no meio urbano: duas do gênero Rattus (Rattus norvegicus ou ratazana, rato de esgoto) e Rattus rattus (ou rato de telhado) e uma do gênero Mus (Mus musculus ou camundongo, rato caseiro). Eventualmente, algumas espécies de gêneros mais adaptados ao ambiente rural ou silvestre, podem invadir as áreas periurbanas e causar danos à população (BRASIL, 1990).

A dificuldade no controle de roedores deve-se ao fato deles serem um dos animais superiores adaptados, capazes de sobreviver e prolíficos por natureza, possuem habilidades de realizar atividades como atravessar frestas, locomoção pelo interior de canos, equilibrar-se sobre cordas e fios, nadar centenas de metros mesmo contra a correnteza, e a capacidade de permanecerem submersos por até três minutos (BRASIL, 1990). Essas habilidades físicas associadas à alimentação

*Recebido em 31 de janeiro de 2021 e aceito em 21 de abril de 2021.

**Médico-Veterinário autônomo, Brasília-DF.

***Diretoria de Vigilância Ambiental em Saúde do Distrito Federal, Brasília-DF.

${ }^{* * \star \star}$ Faculdade de Agronomia e Medicina Veterinária, Universidade de Brasília, Brasília-DF. Autor para correspondência: ligiacantarino@unb.br 
variada conferem uma alta capacidade de adaptação em ambientes urbanos.

Além do desafio permanente contra roedores devido à transmissão de doenças, outro ponto relevante são os prejuízos econômicos relacionados aos alimentos tanto pela ingestão dos mesmos quanto pela contaminação por urina, fezes, e desperdícios por rompimento de sacarias e roeduras parciais de grãos (CARVALHO-NETO, 1987). Os roedores participam da cadeia epidemiológica de pelo menos trinta doenças transmitidas ao homem, sendo a leptospirose uma das mais importantes. As atividades de prevenção e controle de roedores são baseadas no Programa de Vigilância e Controle de Leptospirose, criado em 1987 na Divisão Nacional de Zoonoses, do Ministério da Saúde, visando principalmente o manejo ambiental e controle de roedores, com ênfase na melhoria das condições sanitárias e de moradia da população, minimizando sua exposição ao risco de infecção. A leptospirose é uma doença de notificação compulsória em todo o Brasil, dessa forma, todos os casos suspeitos devem ser notificados à vigilância epidemiológica municipal, por meio de fichas de notificação para inclusão no Sistema Nacional de Agravos de Notificação (Sinan). A notificação é importante, pois permite o registro dos casos e o desencadeamento das medidas de prevenção e controle da doença (BRASIL, 2020).

As atividades da vigilância ambiental estão cada vez mais inseridas nas ações de saúde pelo controle dos animais, pelas intervenções no ambiente e principalmente pelo incremento nas ações educativas, de promoção e conscientização da população de seu importante papel para a modificação do ambiente e, assim aumentar a eficácia das ações da vigilância. Deste modo, estabelecer o controle de roedores eficaz, se constitui em um desafio de importância para a sociedade (VIEIRA, 2012).

Como medida de eliminação são usados agentes raticidas, e seus aplicadores devem estar devidamente munidos de equipamentos de proteção individual (EPIs). Os raticidas possuem mecanismo de ação anticoagulante, agem como antagonista da vitamina $\mathrm{k} 1$, sendo esta necessária para a síntese dos fatores de coagulação II, VII, IX e X, produzidos principalmente no fígado. Além disso, agem diretamente nos vasos, aumentando a permeabilidade vascular no organismo (OGA, 2003). Após ingestão de quantidade suficiente do produto, o roedor apresenta hemorragia interna e externa progressiva até a morte. Atualmente, no Brasil, os únicos rodenticidas permitidos pela Agência Nacional de Vigilância Sanitária (Anvisa) são a base de hidroxicumarina, nas apresentações em forma de pó de contato (usado exclusivamente no interior das tocas), isca granulada e isca ou bloco impermeável (parafinado ou extrusado) (BRASIL, 2016).

É importante conhecer o problema para melhorar as condições ambientais e reduzir a incidência de doenças causadas ou transmitidas por animais sinantrópicos - as zoonoses. Deste modo, é relevante o mapeamento das principais áreas com o problema, de risco ambiental e comunidades vulneráveis (PAPINI, 2012). Explorar a distribuição da ocorrência de queixas e problemas relativos à presença de roedores torna possível verificar estratégias de intervenção das ações de controle de roedores no Distrito Federal, e discutir o emprego, além da aplicação de raticidas e abordagens educativas, de ações integradas com setores de limpeza urbana, infraestrutura e obras, para redução do incômodo e diminuição de doenças associadas aos roedores.

Este trabalho teve por objetivo descrever a distribuição espacial e temporal das reclamações acerca de roedores registradas no setor de animais sinantrópicos da Diretoria de Vigilância Ambiental - DIVAL do Distrito Federal (DF), e possibilitar a discussão de estratégias de intervenção, além do controle populacional, ações integradas com setores de limpeza urbana, infraestrutura e obras, para redução do incômodo e diminuição de doenças associadas aos roedores (BRASIL, 2002).

\section{METODOLOGIA}

O Distrito Federal é uma das 27 unidades federativas do Brasil, sendo a menor e pertencente à Região Centro-Oeste. Por ser um território autônomo, não possui municípios, sendo dividido em 31 regiões administrativas (RAs) e totalizando uma área de $5.779,997 \mathrm{~km}^{2}$, com uma população estimada de 2.977.216 de habitantes (IBGE, 2016). Em seu território, está localizada a capital federal do Brasil, Brasília.

Este estudo descritivo foi realizado com base na análise documental dos registros das reclamações, junto à vigilância ambiental, acerca de problemas relativos à presença de roedores no Distrito Federal, de 2014 a 2016. Os dados foram tabulados e padronizados para o posterior análise, com técnicas da estatística descritiva, empregando-se o software Microsoft Excel ${ }^{\circledR} 2007$ para cálculo da média, desvio-padrão, mediana, além das frequências absoluta e relativa em função do ano de ocorrência, bairro e RA. Com o auxílio do software de estatística Stata ${ }^{\circledR}$ foram realizadas análises de correlação entre a frequência de reclamações e as médias de população, tempo entre a solicitação e o atendimento, forma de solicitação, tipo de logradouro, ambiente público ou privado, foco e estudo socioeconômico por bairro. O software o Sistema de Informação Geográfica Quantum GIS foi utilizado para confecção de mapas.

Os valores, em reais $(R \$)$ de raticidas usados durante as inspeções feitas foram fornecidos pelos técnicos do DIVAL. Os raticidas empregados foram: Maki ${ }^{\circledR}$ bloco extrusado, Bromadiolone 0,005\%, pacote com $1 \mathrm{~kg}-\mathrm{R} \$ 30,00$; Ri do rato ${ }^{\circledR}$ Warfarin, pacote com $1 \mathrm{~kg}-\mathrm{R} \$ 43,00$ e Maki ${ }^{\circledR}$ Pellets parafinados - Bromadiolone 0,005\%, pacote com $1 \mathrm{~kg}$ - R\$35,00. O salário do servidor, considerando o primeiro padrão da primeira classe com base no Anexo I da Lei n 5.237/2013 (GOVERNO DO DISTRITO FEDERAL, 2013), que dispõe da carreira Vigilância Ambiental e Atenção Comunitária à Saúde do quadro de pessoal do Distrito Federal e dá outras providências, foi no valor de $\mathrm{R} \$ 2.485,00$. O preço médio do Diesel foi obtido no Boletim Anual de Preços 2016, referente aos preços do petróleo, gás natural e combustíveis nos mercados nacional e internacional, pela Agência Nacional do Petróleo (BRASIL, 2016 b).

\section{Resultados e discussão}

No período entre 2014 e 2016, na DIVAL/DF e seus Núcleos de Inspeção, foram registradas 10.232 reclamações acerca de roedores. Deste total, $9.375(91,6 \%)$ foram passíveis de avaliação da oportunidade, ou seja o tempo de decorrido entre a solicitação e o atendimento. A oportunidade não pode ser verificada em $8,4 \%$ por ausência da informação de datas nos 
registros da reclamação ou do atendimento. Em 2014, a média da oportunidade foi de 3,48 dias, em 2015; 3,35 dias e, em 2016 , foram 15,61 dias. No triênio, em $90 \%$ das solicitações, a oportunidade foi de até 17 dias.

O maior número de registros de solicitações/reclamações por RA e ano ocorreu em 2015, na RA IX - Ceilândia, com 1.817 reclamações. 64\% (6.602) dos registros das solicitações/reclamações foram para as RAs de Ceilândia, Brasília, Taguatinga e Guará (Figura 1).

Essas três RAs, foram responsáveis pelo maior número de solicitações/reclamações e, as duas primeiras foram as que mais tiveram casos confirmados de leptospirose: 8 e 10 casos, respectivamente. A leptospirose é uma doença infecciosa febril que faz parte da lista de doenças de notificação compulsória no Brasil. Frequentemente é associada à ocorrência de enchentes e inundações, por esses eventos facilitarem a dispersão de ratos, principais vetores urbanos, e de sua urina contaminada por Leptospira (CONFALONIERI et al, 2011; BRASIL, 2020).

O índice pluviométrico foi pouco relevante quando comparado com o número de registros de solicitações/reclamações, pelo contrário, no período em que a precipitação em $\mathrm{mm}$ foi menor, foi maior o número de registro (Figura 2).

A ausência de sazonalidade ocorre pelas espécies de roedores não possuírem período reprodutivo restrito, além da prolificidade e ausência da necessidade de qualquer fator climático para sua ocorrência.

A maioria das ações/atividades para controle dos roedores foram em decorrência de solicitações/reclamações registradas via telefone. Porém com a dificuldade na comunicação motivada pela ausência de tal a partir do final de 2015, as reclamações reduziram de 4.429 em 2014 para 1.358 no ano de 2016 (Tabela 1).

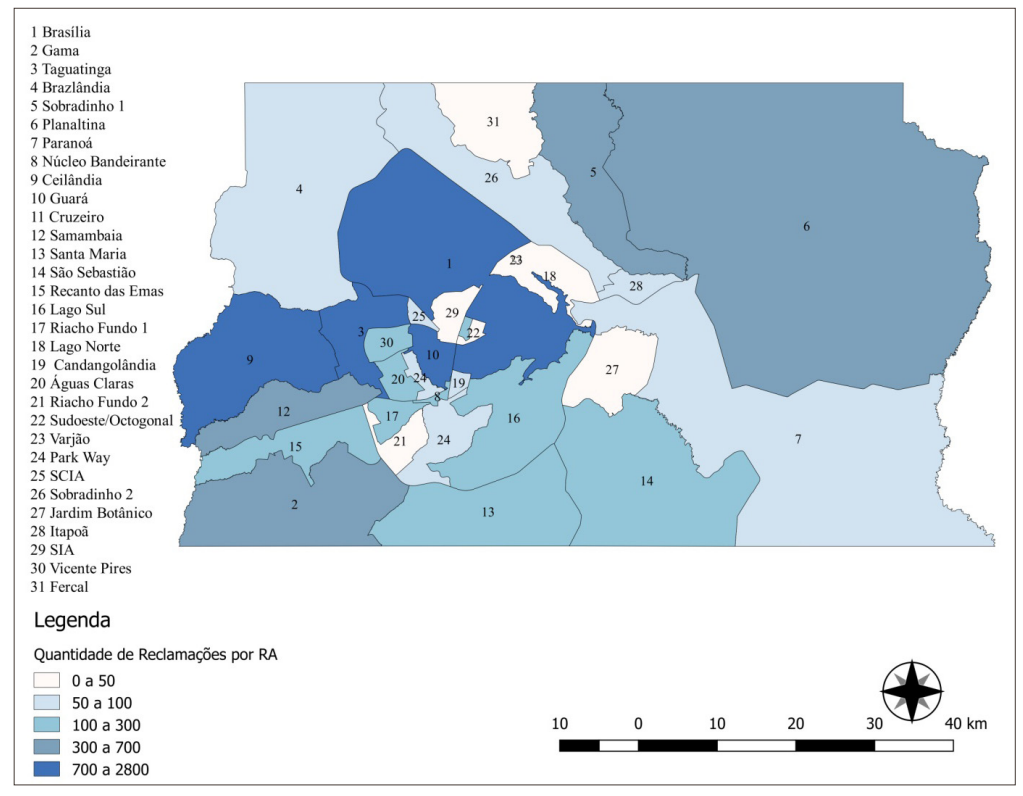

Figura 1: Distribuição dos registros das solicitações/reclamações acerca de roedores no DF, 2014-2016

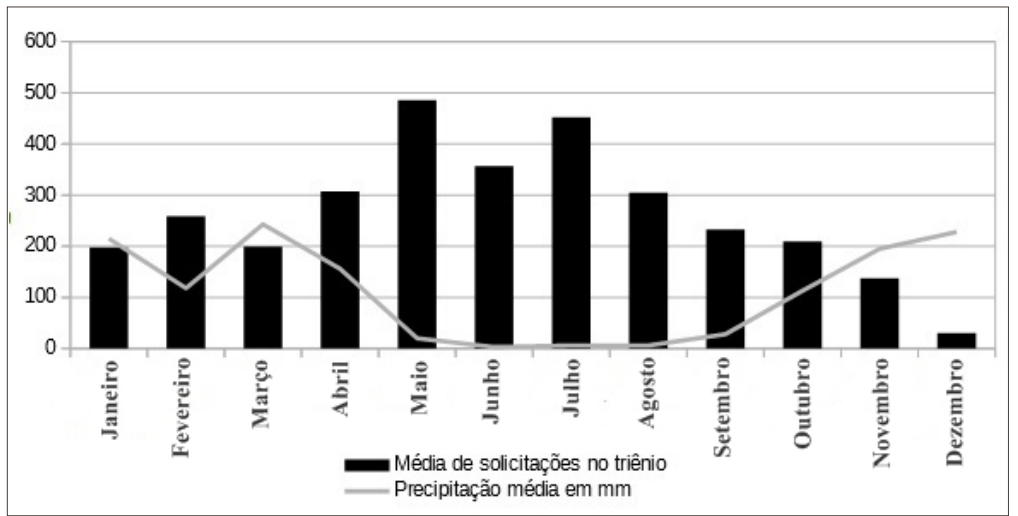

Figura 2: Variação da frequência média de reclamações sobre roedores, entre 2014-2016 e da precipitação média histórica, por mês 
Tabela 1: Registro de formas de solicitações/reclamações acerca de roedores no DF, 2021-2017

\begin{tabular}{lcccc}
\hline \multirow{2}{*}{ Forma de Solicitação } & \multicolumn{3}{c}{ Ano } & \multirow{2}{*}{ Total } \\
\cline { 2 - 4 } & 2014 & 2015 & 2016 & \\
\hline Demanda ativa & 1308 & 1897 & 109 & 3314 \\
Denúncia & 35 & 59 & 34 & 128 \\
E-mail & 16 & 1 & 12 & 29 \\
Memorando & 0 & 4 & 1 & 5 \\
Ofício & 213 & 89 & 124 & 426 \\
Telefone & 2191 & 1293 & 0 & 3484 \\
Ouvidoria & 87 & 140 & 103 & 330 \\
Pessoalmente & 120 & 128 & 202 & 450 \\
Outros & 459 & 759 & 773 & 1991 \\
Total & 4.429 & 4.370 & 1.358 & 10.157 \\
\hline
\end{tabular}

De todas as solicitações/reclamações, $9,8 \%$ foram provenientes de ambientes públicos e, consequentemente, 90,2\% (9.218) de ambientes privados. Desses, $67 \%$ (6.902) foram de residências, sendo justificado, pela população ansiando por desratização gratuita. $12 \%$ foram para estabelecimentos comerciais, exteriorizando a impressão de limpeza para seus usuários. Nos locais públicos, entre as 1.001 solicitações/reclamações, 480 foram para escolas, o que representa cerca de $48 \%$ (Tabela 2).

Quanto à presença de vestígios de roedores nas vistorias realizadas no período, foram encontradas 21.674 trilhas e 8.475 tocas de roedores, e empregados toneladas de raticidas em pó $(1,232)$, blocos (13.601) e iscas (50.088), que foram dirigidos, principalmente, para edificações privadas ou particulares.

Deve ser considerado que nas vistorias realizadas motivadas por essas solicitações/reclamações, em pouco mais da metade, foram identificados alguns indícios de roedores ou foco, como manchas de gordura, fezes, trilhas, tocas, roeduras, entre outros (Tabela 2).

Houve correspondência entre o número de solicitações/ reclamações e o tamanho da população residente nas RAs, mas o mesmo não ocorreu ao analisar o grau de escolaridade e a renda per capita, características que pouco influenciaram na quantidade de reclamações (Figura 3). As RAs de Samambaia e Brasília, com renda média per capita de $\mathrm{R} \$ 914,61$ e $\mathrm{R} \$ 5.559,75$, respectivamente, apresentaram números de 0,38 quando calculados os valores relativos.

Quando analisados os valores relativos (Equação 1), houve destaque para as regiões do Setor de Indústria e Abastecimentos - SIA e de Brasília. Outro importante fator a ser observado ao considerar o número de solicitações e a população, é o número de estabelecimentos comerciais. A RA com maior valor relativo, foi a do SIA, que agrega um grande número de concessionárias de automóveis, motocicletas e náutica. A despeito das instalações, que transparecem higiene para os consumidores, 0 número da população (1.990) influenciou enviesando o resultado. $\mathrm{Na}$ sequência foi a RA 1- Brasília, com 210.067 habitantes, 2.108 solicitações e 1.327 focos (com vestígios de roedores), com o valor relativo de 6.31 causado por sua extensão, grau de escolaridade elevado e proximidade com fatores influentes, como pontos turísticos, sede dos governos distrital e da República.

Tabela 2: Tipos de logradouros constantes nos registros solicitações/reclamações que requisitam serviço de controle de roedores (desratização) no DF, 2014-2016

\begin{tabular}{|c|c|c|c|}
\hline Tipo de Logradouro & Geral & Presença de focos & $\%$ \\
\hline Casa residencial & 6902 & 3524 & 51,1 \\
\hline Estabelecimento comercial & 1258 & 709 & 56,4 \\
\hline Apartamento residencial & 510 & 279 & 54,7 \\
\hline Escola & 480 & 301 & 62,7 \\
\hline Órgão público & 371 & 187 & 50,4 \\
\hline Chácara & 194 & 42 & 21,6 \\
\hline Condomínio & 165 & 124 & 75,2 \\
\hline Terreno baldio & 97 & 16 & 16,5 \\
\hline Via pública & 76 & 25 & 32,9 \\
\hline Área & 33 & 8 & 24,2 \\
\hline Feira & 29 & 17 & 58,6 \\
\hline Igreja & 21 & 13 & 61,9 \\
\hline Unidade de Saúde & 18 & 9 & 50,0 \\
\hline Creche & 15 & 9 & 60,0 \\
\hline Outros & 14 & 5 & 35,7 \\
\hline Asilo & 10 & 4 & 40,0 \\
\hline Hípica & 10 & 3 & 30,0 \\
\hline Lago & 2 & 0 & 0,0 \\
\hline Praça pública & 2 & 0 & 0,0 \\
\hline Assentamento & 1 & 1 & 100,0 \\
\hline Clube & 1 & 0 & 0,0 \\
\hline Oficina & 1 & 0 & 0,0 \\
\hline Parque & 1 & 0 & 0,0 \\
\hline Posto Policial & 1 & 0 & 0,0 \\
\hline Geral & 10.212 & 5.276 & 51,7 \\
\hline
\end{tabular}




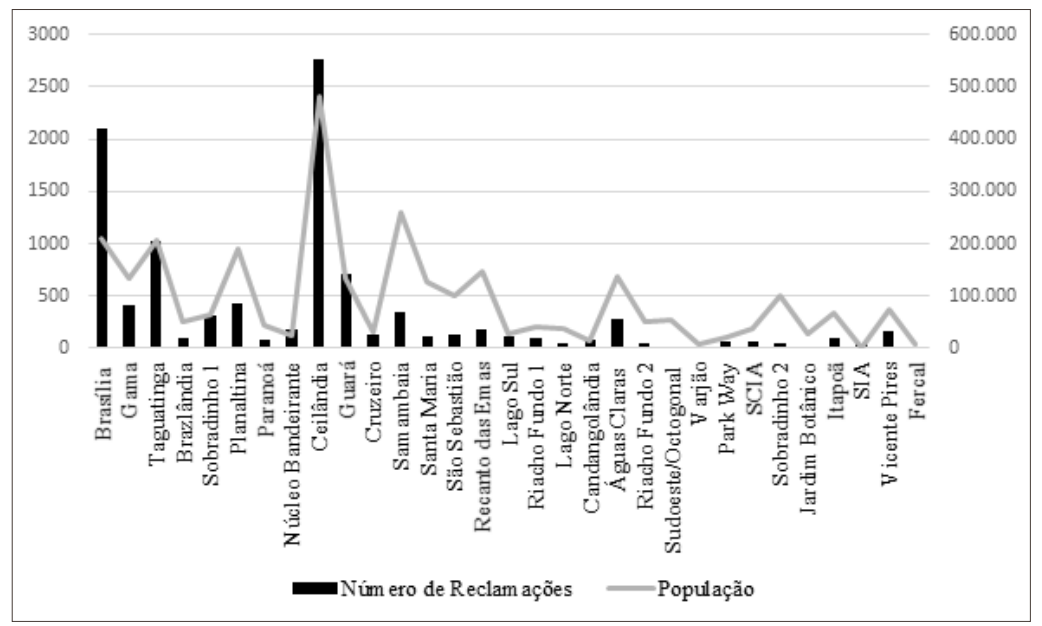

Figura 3: Frequência de solicitações/reclamações acerca roedores, por população e RA no DF, 2014-2016

$$
\text { Valor relativo }=\frac{\text { População }}{1.000} \times n^{\circ} \text { de focos }
$$

\section{Equação 1}

$\mathrm{Na}$ observação de ocorrências e atendimentos às solicitações em residências privadas, e os fatores básicos, como salário do servidor, gastos com transporte (diesel apenas) e os valores dos produtos químicos, é possível mensurar o gasto com funcionários (Equação 2). Há que se considerar para gestão dos recursos financeiros a remuneração do servidor, a carga horária, o número de atendimentos a residências particulares de 6.902, e multiplicado por 2, por ser a quantidade mínima necessária de servidores. Estipulando a distância de $15 \mathrm{~km}$ para ida e volta aos locais solicitados, considerando que em cada regional e adjacência, a quilometragem por litro $(10 \mathrm{~km} / \mathrm{L})$, o preço médio do combustível (diesel) nos 3 anos e o combustível utilizado.

$\mathrm{Na}$ integralidade das residências inspecionadas, em 3.524 haviam ao menos uma caixa de passagem de esgoto com vestígio de roedor (marcha de gordura, fezes, arranhaduras, mordeduras), 1.073 apresentavam ao menos uma toca e 760 continham trilhas. Como medida de controle, são empregados três blocos por caixa com algum vestígio, 50 gramas de pó por toca e 25 gramas de isca em cada trilha, respectivamente. Os raticidas utilizados foram nas quantidades de 211 pacotes de blocos extrusados, $54 \mathrm{~kg}$ de pó de contato e 19 de iscas.

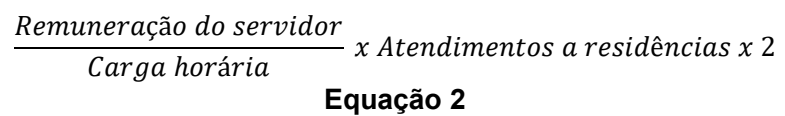

Foram notificados 11.901 casos confirmados de leptospirose no Brasil nos anos estudados e destes, 82 ocorreram no DF. $\mathrm{Na}$ hipótese de que a metade dos casos foram hospitalizados, e a outra parte recebeu tratamento ambulatorial, levando em consideração os valores apresentados por Pereira (2013) - de $\mathrm{R} \$ 603,63$ para casos hospitalares e $\mathrm{R} \$ 15,43$ para a casos ambulatoriais -, apenas com custos médicos envolvendo internação, consultas médicas, medicamentos, exames complementares e remuneração dos profissionais, o custo médio sobre tais variáveis chega a valores consideráveis para serem gastos apenas com o suporte médico. Além destes, os custos não médicos (cuidados familiares, transporte dos doentes e dos parentes para as unidades de saúde), custos indiretos (redução da produtividade de resultantes de alterações no estados de saúde - morbidade e mortalidade -, tempo de lazer perdido, tempo perdido por familiares - visitas e acompanhamento hospitalar), ademais custos intangíveis, englobando aspectos físicos, emocionais e sociais (perda de bem-estar, dor e desconforto) também devem ser considerados numa avaliação sob a ótica da economia da saúde.

\section{Considerações finais}

Em relação a outros animais sinantrópicos, os roedores são vistos como vilões e provocam repugnância na população por suas características, grandes perdas econômicas e danos materiais, além da capacidade de transmissão de doenças.

O intuito do controle de roedores é evitar custos e mesmo que a prevenção tenha custo, certamente é menor do que o do atendimento, e o bem-estar da sociedade deve sempre estar em destaque, o que evidencia a necessidade de desenvolver meios capazes de diminuir, além dos custos, a incidência, a gravidade e a letalidade de problemas relacionados à saúde como a leptospirose. Mas para que isso ocorra é preciso mais que os esforços isolados do setor de saúde: é fundamental a gestão integrada entre os diversos setores da gestão pública, uma vez que não são apenas as medidas específicas em saúde que podem levar a alterações no comportamento da doença.

Houve correspondência entre o número de solicitações/ reclamações e o tamanho da população residente nas RAs, no entanto, renda e grau de escolaridade influenciaram pouco no número de registro de queixas reclamações. A variável que mais influenciou no número de notificações foi a densidade demográfica. Renda per capita e grau de escolaridade não foi representativa.

Quanto à presença de vestígios detectadas nas vistorias realizadas no período chama atenção à utilização de toneladas de raticidas, dirigidos, principalmente a edificações privadas ou particulares. Esta situação alerta para o debate da responsabilidade partilhada no controle de animais sinantrópicos: o Estado, gastando recursos que poderiam ser investidos de outras formas - considerando ainda tempos de crise - ou a população e a gestão pública, que não dão devido valor aos setores de infraestrutura e limpeza urbana?

Alguns desafios encontrados durante o desenvolvimento do trabalho foram a incongruência de dados, o preenchimento incompleto dos relatórios de vistoria e inspeção, a dificuldade na comunicação entre a população e o órgão, o que reduziu drasticamente os números de solicitações/reclamações.

As ações que envolvam de comunicação e educação em saúde são importantes no controle de roedores e outros animais sinantrópicos e devem ser preconizadas no desenvolvimento de políticas públicas integradas entre serviços públicos e sociedade civil em prol da saúde humana, animal e ambiental, na constituição da saúde única. 


\section{Agradecimentos}

Os autores agradecem o incentivo do Programa Institucional de Bolsas de Iniciação Científica (2017) da Universidade de Brasília e ao corpo técnico da Diretoria de Vigilância Ambiental (DIVAL/DF) pelo apoio no desenvolvimento do trabalho.

\section{Referências}

BRASIL. Ministério da Saúde. Fundação Nacional de Saúde. Coordenação de Controle de Zoonoses e Animais Peçonhentos. Normas Operacionais de Centros de Controle de Zoonoses Procedimentos para o Controle de Populações Animais Urbanos. Fundação Nacional de Saúde. CENEPI - Centro Nacional de Epidemiologia, 1990.

BRASIL. Fundação Nacional de Saúde. Manual de controle de roedores. Brasília: Ministério da Saúde, 2002. 132p.

BRASIL. Ministério da Saúde. Secretaria de Atenção à Saúde. Departamento de Atenção Básica. Vigilância em saúde: zoonoses / Ministério da Saúde, Secretaria de Atenção à Saúde, Departamento de Atenção Básica. Brasília: Ministério da Saúde, 2009.

BRASIL. Ministério da Saúde. Secretaria de Vigilância em Saúde. Departamento de Vigilância das Doenças Transmissíveis. Manual de vigilância, prevenção e controle de zoonoses: normas técnicas e operacionais. Brasília: Ministério da Saúde, 2016.

BRASIL. Agência Nacional do Petróleo, Gás Natural e Biocombustíveis (ANP). Boletim Anual de Preços: preços do petróleo, gás natural e combustíveis nos mercados nacional e internacional. Rio de Janeiro: ANP, 2016 (b). Disponível em: < http://www.anp.gov.br/images/Boletim-Anual/Boletim-2016.pdf>. Acesso em 15 mai 2021.

BRASIL. Ministério da Saúde. Gabinete do Ministro. Portaria $n^{\circ} 264$, de 17 de fevereiro de 2020. Altera a Portaria de Consolidação $n^{\circ}$ 4/GM/MS, de 28 de setembro de 2017, para incluir a doença de Chagas crônica, na Lista Nacional de Notificação Compulsória de doenças, agravos e eventos de saúde pública nos serviços de saúde públicos e privados em todo o território nacional. Disponível em: https://bvsms.saude.gov.br/ bvs/saudelegis/gm/2020/prt0264_19_02_2020.html. Acesso em 15 mai 2021.
CARVALHO NETO, C. Manual prático da biologia e controle dos roedores. São Paulo: Ciba-Geigy, 1987.

CONFALONIERI, U.E.C. Vulnerabilidades em matéria de saúde pública na Região Metropolitana do Rio de Janeiro na perspectiva das mudanças climáticas. In: LAGET (ed). Megacidades, Vulnerabilidades e Mudanças Climáticas: região metropolitana do Rio de Janeiro. Rio de janeiro: LAGET, 2011. p.201-225. Disponível em: <http://www.poli.ufrj.br/noticias/arquivos/completo. pdf>. Acesso em: 15 jul 2017.

GOVERNO DO DISTRITO FEDERAL. Lei n 5.237 , de 16 de dezembro de 2013. Dispõe sobre a carreira Vigilância Ambiental e Atenção Comunitária à Saúde do Quadro de Pessoal do Distrito Federal e dá outras providências. Disponível em: < http://www. sinj.df.gov.br/sinj/Norma/75947/Lei_5237_16_12_2013.html>. Acesso em 10 mai 2021.

IBGE. Fundação Instituto Brasileiro de Geografia e Pesquisa. Cidades - Distrito Federal: Informações Completas, 2016. [online] Disponível em: <http://cod.ibge.gov.br/904>. Acesso em: 29 jul 2017.

OGA, S. Fundamentos de Toxicologia. Atheneu Editora, São Paulo, p 437-458, 2003.

PAPINI, S. Vigilância em saúde ambiental: uma nova área da ecologia. $2^{a}$ ed.rev. e amp. São Paulo: Atheneu Editora, 2012. PEREIRA, C. A. R. Custo social da leptospirose no Brasil e o efeito de chuvas extremas em Nova Friburgo para o incremento de casos da doença. Dissertação (Mestrado). Escola Nacional de Saúde Pública Sergio Arouca, Rio de Janeiro, 2013.

VIEIRA, K. P.B. A. Reestruturação das Ações de Controle de Roedores no Município do Jaboatão dos Guararapes - PE. Monografia (Especialização em Gestão de Sistemas e Serviços de Saúde). Departamento de Saúde Coletiva, Centro de Pesquisas Aggeu Magalhães, Fundação Oswaldo Cruz, Recife. 2012, $30 \mathrm{p}$. 